\title{
Optimization of Design Parameters for Spines of Various Geometries
}

Rong-Hua Yeh

Department of Marine Engineering and Technology National Taiwan Ocean University Keelung, Taiwan, R. O. C.

Follow this and additional works at: https://jmstt.ntou.edu.tw/journal

Part of the Engineering Commons

\section{Recommended Citation}

Yeh, Rong-Hua (1995) "Optimization of Design Parameters for Spines of Various Geometries," Journal of Marine Science and Technology. Vol. 3: Iss. 1, Article 3.

DOI: 10.51400/2709-6998.2501

Available at: https://jmstt.ntou.edu.tw/journal/vol3/iss1/3

This Research Article is brought to you for free and open access by Journal of Marine Science and Technology. It has been accepted for inclusion in Journal of Marine Science and Technology by an authorized editor of Journal of Marine Science and Technology. 


\title{
OPTIMIZATION OF DESIGN PARAMETERS FOR SPINES OF VARIOUS GEOMETRIES
}

\author{
Rong-Hua Yeh \\ Department of Marine Engineering and Technology \\ National Taiwan Ocean University \\ Keelung, Taiwan, R. O. C.
}

Key words: optimization, spine, power-law.

\begin{abstract}
This work presents a systematic study on the optimum base diameter, length, heat dissipation, temperature profile, and efficiency of spines by employing fin parameter versus fin efficiency method. The temperature dependent heat transfer coefficient is assumed to be a power-law type. Several common fin geometries including cylindrical, convex parabolic, conical, and concave parabolic profiles are investigated respectively. It is found that the optimum dimensions of spines are functions of fin volume, heat transfer coefficient at fin base, and thermal conductivity. To facilitate the thermal design of heat transfer components, simple mathematical expressions as well as design charts are presented.
\end{abstract}

\section{INTRODUCTION}

Applications for finned surfaces are widely seen in air- conditioning, aircraft, vehicles, and vessels. Since weight and material costs are the primary design considerations in most of these applications, it is highly desirable to obtain the optimum design information of fins.

The information of optimum design of extended surfaces is - readily available in the work of Kern and Kraus [1]. Recently, a thorough review for fin technology has been presented by Kraus [2]. For spines, Sonn and Barr-Cohen [3] and $\mathrm{Li}$ [4] dealt with the optimization of a single cylindrical fin and pin fin array respectively. Considering convective spines of cylindrical, convex parabolic, conical, and concave parabolic profiles with curvature effect, Chung et al. [5] have presented a systematic study on the optimum dimensions for these fins. As for radiative heat transfer, Chung and Nguyen [6] formulated the general relationships for spine dimensions as well as heat transfer characteristics under the optimum condition. In addition, the optimum geometry of the fin with least weight is uniquely determined in their work.

For boiling heat transfer from fins, the heat transfer coefficient can be expressed as an empirical function of the temperature difference between the fin surface and liquid. In the fin optimization problems, Haley and Westwater [7], used a numerical computation to find out a turnip-shape-spine that would dissipate heat at smallest volume. Yeh and Liaw [8] proposed the optimum excavated shape in a cylindrical fin with minimum weight. Employing a temperature correlated profile, Razani and Zohoor [9] and Sohrabpour and Razani [10] investigated the optimization of fins with temperature dependent heat transfer coefficient. Very recently, using an integral approach, Chung and Iyer [11] have determined the optimum dimensions for longitudinal rectangular fins and cylindrical pin fins by incorporating transverse heat conduction. Due to the complexity of their results, only numerous design charts are given.

From the foregoing descriptions, it is apparent that most works regarding boiling heat transfer are dealing with optimum fin shape. To the best of the author's knowledge, there has not been any paper published to quantitatively analyze the optimum characteristics of fins in boiling as well as natural convective heat transfer. In this study, for a given cylindrical, convex parabolic, conical, and concave parabolic profiles of fins, the fin base diameter and fin length which maximize the heat duty for a fixed volume are investigated. Considering a powerrlaw type heat transfer coefficient, simple formulas for the base diameter, length, heat dissipation, temperature distributions and efficiency of the optimum spines are obtained. In addition to the laminar as well as turbulent free convection, the results of this study can also be applied to nucleate and film boiling heat transfer modes. 


\section{MATHEMATICAL ANALYSIS}

A spine with an arbitrary profile is now considered. The surface heat flux along the fin length varies following a powerr law type dependence on the temperature difference between the fin and the ambient fluid, i.e.

$$
q=a \theta^{m}
$$

where $a$ and $m$ are dimensional and dimensionless constants respectively. The values of $a$ and $m$ depend on the boiling liquids and the types of heat transfer. For instance, the exponent $m$ may take the values of 0.75 , $1,1.25,1.33,3$, and 4 depending on whether the heat transfer process dominates by film boiling, forced convection, laminar free convection, turbulent free convection, nucleate boiling, or radiation into free space at zero temperature. Note that the case $m=1$ corresponds to the "linear" cooling process. In this situation, $a$ is equivalent to the constant heat transfer coefficient, $h$. As for $m=4, a$ stands for the product of emissivity, $\epsilon$, of the fin material and Stefan-Boltzmann constant, $\sigma$.

Throughout this analysis, a few assumptions are made as follows: (1) The material of the fin is homogeneous and the thermal conductivity of the fin is constant; (2) no heat source or sink exists within the fin; (3) the ambient fluid temperature is maintained at a constant value, $T_{a}$; (4) There is no bond resistance at the base and the base temperature, $T_{b}$, is uniform. The general fin profile function can be expressed by

$$
f_{1}(x)=\frac{D}{2}\left(\frac{x}{l}\right)^{n}
$$

where $D$ and $l$ denote the base diameter and length of the fin and the profile index represents

$\begin{array}{ll}\text { cylindrical profile } & \text { for } n=0 \\ \text { convex parabolic profile } & \text { for } n=0.5 \\ \text { conical profile } & \text { for } n=1 \\ \text { concave parabolic profile } & \text { for } n=2\end{array}$

The volume of spines may be written in terms of base diameter and length and is given by $V=\pi D^{2} l /[4(2 n+1)]$. Applying the aforementioned assumptions and substituting the general fin profiles, the one-dimensional steady state heat conduction equation inside the fin can be described by the following equation

$$
\frac{d}{d x}\left(x^{2 n} \cdot \frac{d \theta}{d x}\right)-\frac{4 a}{k D}(l x)^{n} \cdot \theta^{m}=0
$$

where $k$ is the thermal conductivity of the fin. The terminology and coordinate system are shown in Fig. 1. Note that the origin of the coordinate system is taken at fin tip and positive $x$ is toward the fin base. Introducing the non-dimensional parameters, $X=x / l, f(X)=\theta / \theta_{b}$, and $N=4 h_{b} l^{2} /(k D)$, equation (3) may be rewritten in the form

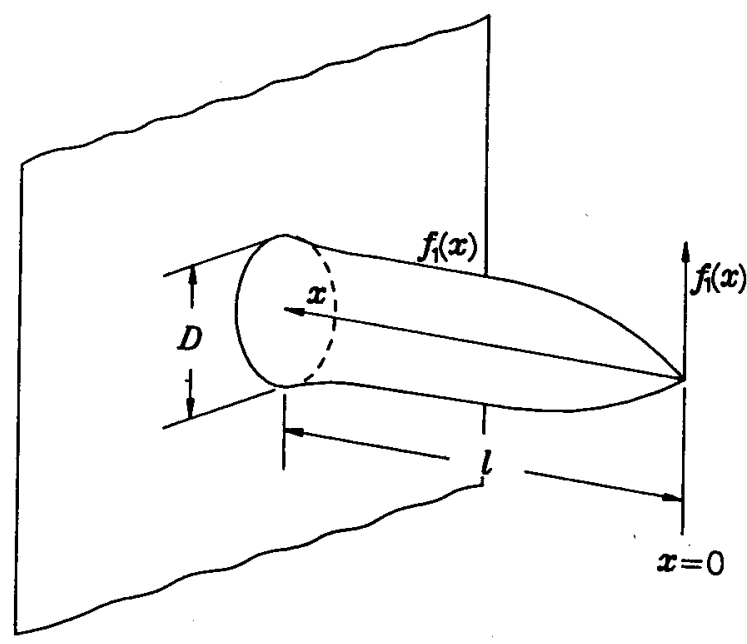

Fig. 1. Schematic diagram of the spine.

$$
\frac{d}{d X}\left(x^{2 n} \cdot \frac{d f}{d X}\right)-N X^{n} f^{m}=0
$$

It is noted that $h_{b}\left(=a \theta_{b}^{m-1}\right)$ is the heat transfer coefficient at fin base.

\section{Spine of cylindrical profile $(n=0)$}

Consider negligible heat loss from the free end, the heat flow through the fin base can be evaluated from the equation below

$$
Q=\left(16 \pi^{2}\right)^{1 / 5} \cdot \theta_{b} \cdot\left(h_{b}{ }^{4} k V^{3}\right)^{1 / 5} \cdot N^{-4 / 5} \cdot f^{\prime}(1)
$$

The spine efficiency is defined as the ratio of the actual heat dissipated by the fin to the ideal heat transferred if the entire spine is operating at the base temperature. Hence the efficiency of the optimum fin can be expressed as

$$
\eta=f^{\prime}(1) / N
$$

Substituting equation (6) into (5) gives

$$
Q=\left(16 \pi^{2}\right)^{1 / 5} \cdot \theta_{b} \cdot\left(h_{b}{ }^{4} k V^{3}\right)^{1 / 5} \cdot N^{1 / 5} \cdot f^{\prime} \cdot \eta
$$

For known values of $\theta_{b}, k$, and $V$, a relationship between $N$ and $\eta$ can be obtained to maximize $Q$. A detailed solution method is described in the end of this section. Note that $N$ may also be referred as fin parameter. A certain value of $N$ which leads to the maximum heat dissipation of a fin is designated as $N_{o p t}$. Hence, the dimensionless optimum base diameter and length of the fin become 


$$
\begin{aligned}
& D^{*}=\frac{D_{o p t}}{\left(\frac{h_{b} V^{3}}{k}\right)}=\left[64 /\left(\pi^{2} N_{o p t}\right)\right]^{1 / 5} \\
& l^{*}=\frac{l_{o p t}}{\left(\frac{k^{2} V}{h_{b}{ }^{2}}\right)^{1 / 5}}=\left[N_{o p t}^{2} /(4 \pi)\right]^{1 / 5}
\end{aligned}
$$

and the maximum heat dissipation is obtained as

$$
Q^{*}=\frac{Q_{o p t}}{\theta_{b}\left(h_{b}^{4} k V^{3}\right)^{1 / 5}}=\left(16 \pi^{2} / N_{o p t}^{4}\right)^{1 / 5} \cdot f^{\prime}(1)
$$

The fin efficiency of the optimum fin is the same as that given in equation (6) with $N_{\text {opt }}$ instead of $N$.

\section{Spine of convex parabolic profile $(n=0.5)$}

In this case, the boundary condition at fin tip is the same as previous case. The heat duty of this spine in terms of fin parameter and efficiency are derived as

$$
Q=\frac{4}{3}\left(4 \pi^{2}\right)^{1 / 5} \theta_{b} \cdot\left(h_{b}^{4} k V^{3}\right)^{1 / 5} \cdot N^{1 / 5} \cdot \eta
$$

Similarly, an optimum $N$ may be obtained to maximize $Q$. The dimensionless quantities are defined in equations (8)-(10) and are calculated as

$$
\begin{aligned}
& D^{*}=\left[256 /\left(\pi^{2} N_{o p t}\right)\right]^{1 / 5} \\
& l^{*}=\left[N_{o p t}^{2} /(2 \pi)\right]^{1 / 5} \\
& Q^{*}=\left(128 \pi^{2} / N_{o p t}^{4}\right)^{1 / 5} \cdot f^{\prime}(1)
\end{aligned}
$$

and

$$
\eta=1.5 f^{\prime}(1) / N_{o p t}
$$

\section{Spine of conical profile $(n=1)$}

The solution of this fin exists only when a finite temperature excess (superheat) is imposed at the free end. The total heat transfer rate of a conical fin is expressible as

$$
Q=\left(\frac{27}{2} \pi^{2}\right)^{1 / 5} \theta_{b} \cdot\left(h_{b}{ }^{4} k V^{3}\right)^{1 / 5} \cdot N^{1 / 5} \cdot \eta
$$

Similar to previous cases, for a fixed $\theta_{b}, k$, and, $V$, the total heat duty $Q$ may be maximized by choosing an appropriate $N_{\text {opt }}$. The dimensionless optimum dimen- sions, heat dissipation, and efficiency of this spine thus becomes

$$
\begin{aligned}
& D^{*}=\left[576 /\left(\pi^{2} N_{o p t}\right)\right]^{1 / 5} \\
& l^{*}=\left[3 N_{o p t}^{2} /(4 \pi)\right]^{1 / 5} \\
& Q^{*}=\left(432 \pi^{2} / N_{o p t}^{4}\right)^{1 / 5} \cdot f^{\prime}(1)
\end{aligned}
$$

and

$$
\eta=2 f^{\prime}(1) / N_{o p t}
$$

\section{Spine of concave parabolic profile $(n=2)$}

In view of Eq. (4), this fin exists only when the tip temperature is equal to the environmental fluid. The heat dissipation, $Q$, may be expressed in terms of $N$ and $\eta$, i.e.

$$
Q=\frac{\left(2000 \pi^{2}\right)^{1 / 5}}{3} \cdot\left(h_{b}{ }^{4} k V^{3}\right)^{1 / 5} \cdot \theta_{b} \cdot N^{1 / 5} \cdot \eta
$$

The optimum base diameter, length, heat dissipation, and efficiency of a fin are then calculated from the following relationships

$$
\begin{aligned}
& D^{*}=\left[1600 /\left(\pi^{2} N_{o p t}\right)\right]^{1 / 5} \\
& l^{*}=\left[5 N_{o p t}^{2} /(4 \pi)\right]^{1 / 5} \\
& Q^{*}=\left(2000 \pi^{2} / N_{o p t}^{4}\right)^{1 / 5} \cdot f^{\prime}(1)
\end{aligned}
$$

and

$$
\eta=3 f^{\prime}(1) / N_{o p t}
$$

\section{Solution procedures}

By letting $d Q=0$, the maximum heat dissipation can be achieved and one obtain

$$
\eta=N^{-1 / 5}
$$

Imposing appropriate given boundary conditions at tips, Eq. (4) is solved by finite difference method with linearized source term [12]. An alternative way to solve Eq. (4) may be done by the fourth order Runge-Kutta method. The fin efficiency, $\eta$, is evaluated for each given $N$. A logarithmic coordinates is used in plotting versus $N$. The optimum design, $N=N_{\text {opt }}$, is reached when the slope of the curve is $-1 / 5$. Once $N_{\text {opt }}$ and $f^{\prime}(1)$ 


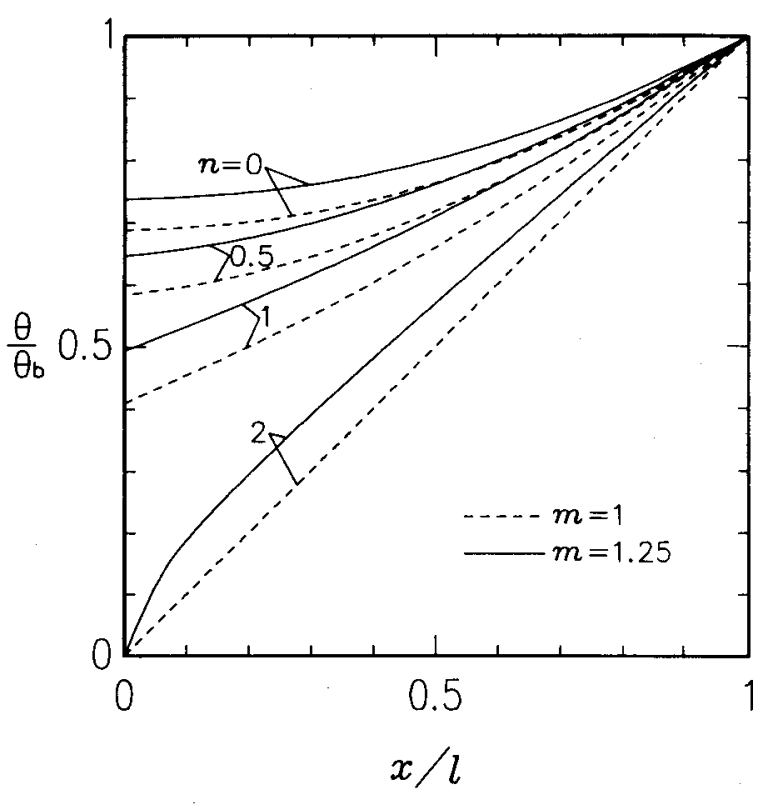

Fig. 2. Dimensionless temperature profiles of spines for $m=1$ and 1.25 .

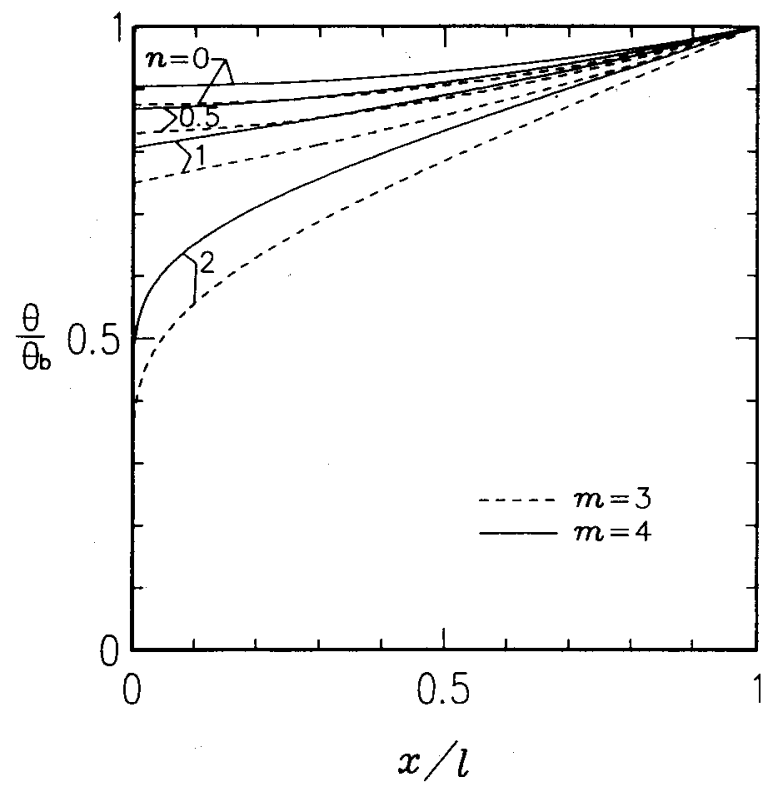

Fig. 3. Dimensionless temperature profiles of spines for $m=3$ and 4 .

are evaluated, the optimum data can then be readily obtained.

\section{RESULTS AND DISCUSSION}

For spines with cylindrical, convex parabolic, conical, and concave parabolic profiles, the optimum base diameter, length, temperature distribution, heat duty, and efficiency of the fins are obtained in many heat

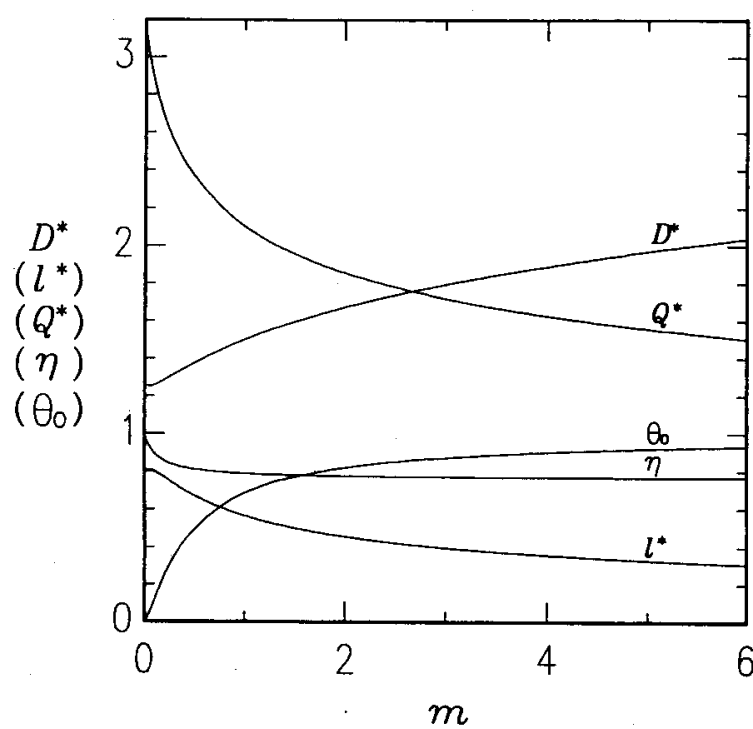

Fig. 4. Dimensionless optimum base diameter, length, heat duty, tip temperature, and efficiency for a cylindrical pin fin.

transfer modes. In this study, two variables, fin parameter and fin efficiency, are adopted in the fin optimization problem for boiling heat transfer. The optimum dimensions and heat transfer characteristics of a fin totally depend on fin volume, heat transfer coefficient at fin base, and thermal conductivity of spines for a given fin profile.

Figure 2 shows the dimensionless temperature distributions of the optimum spines in both forced and natural convection. It is interesting to learn that the temperature profile of a fin with a given concave parabolic fin profile, $n=2$, is concave downward for $m=1.25$ whereas a linear temperature profile [1] is seen for a least-material fin whose shape also turns out to be a concave parabolic profile for $m=1$. In addition, the temperature is a little higher at a larger $m$. Similar trends in temperature profiles are found in Fig. 3 at $m=3$ and $m=4$.

In boiling heat transfer, the values of $m$ may vary from 2 to 5.5 as indicated in the works of Dhir and Liaw [14] and Gaertner [15] for nucleate boiling and $0<m<$ 1 for film boiling [7] or film condensation. Figures 4-7 display the base diameter, length, heat duty, and efficiency for optimum spines of cylindrical, convex parabolic; conical, and concave parabolic profiles for $0 \leq$ $m \leq 6$. As a whole, fin efficiency decreases abruptly with increasing $m$ for $m<1$ and changes slightly for $2 \leq$ $m$. For a conical and a concave-parabolic-profile fins, it is noted that no optimum design is found as $m$ is close to zero. This is due to the fact that the dimensionless temperature drops to zero in the middle section of the fin in a long fin for $0<m<1$. This result is similar to 


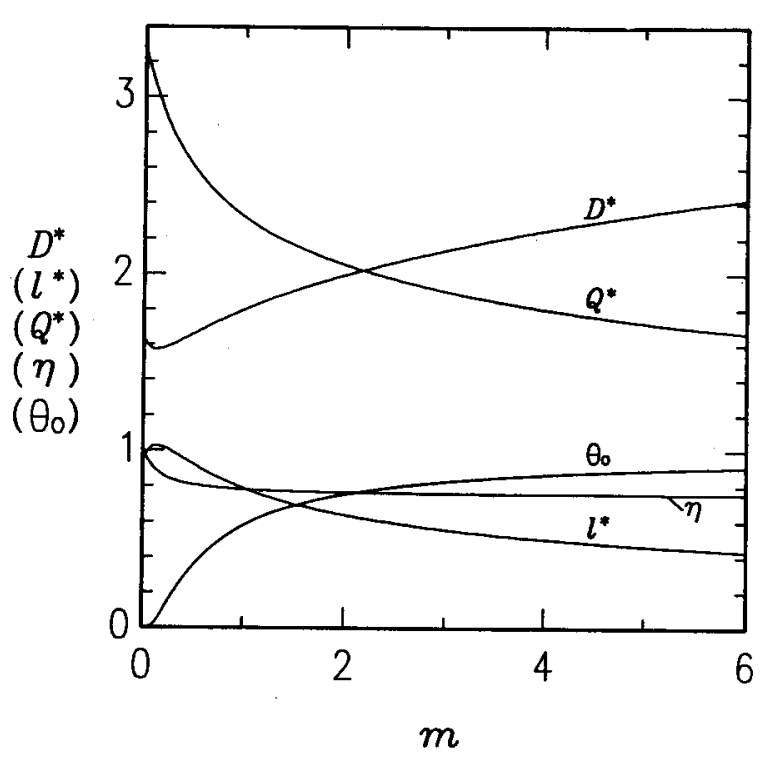

Fig. 5. Dimensionless optimum base diameter, length, heat duty, tip temperature, and efficiency for a spine of convex parabolic profile.

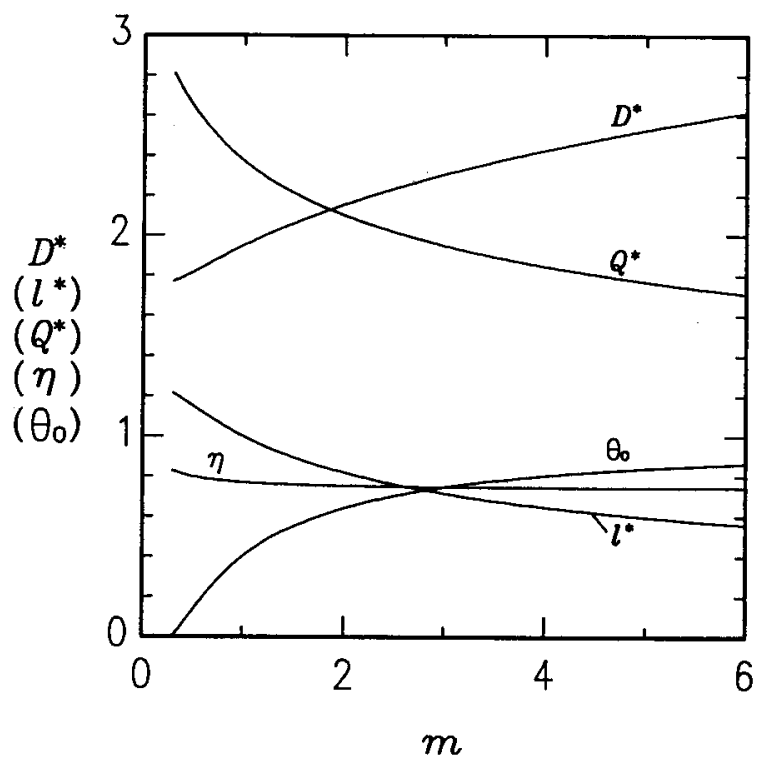

Fig. 7. Dimensionless optimum base diameter, length, heat duty, and efficiency for a spine of concave parabolic profile.

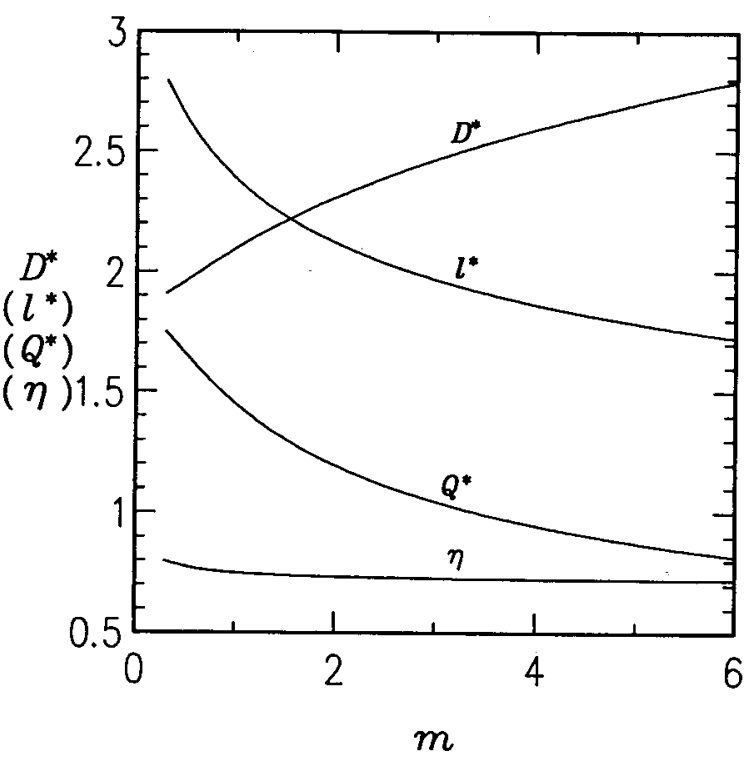

Fig. 6. Dimensionless optimum base diameter, length, heat duty, tip temperature, and efficiency for a conical spine.

Table 1. Dimensionless optimum base diameter, length, heat duty, efficiency, and tip temperature of cylindrical pin fins for $m=0.75,1,1.25,1.33,3$, and 4 .

\begin{tabular}{cccccc}
\hline$m$ & $D^{*}$ & $l^{*}$ & $Q^{*}$ & $\eta$ & $\theta_{o}$ \\
\hline 0.75 & 1.446 & 0.609 & 2.205 & 0.797 & 0.617 \\
1 & 1.503 & 0.564 & 2.100 & 0.789 & 0.688 \\
1.25 & 1.553 & 0.528 & 2.020 & 0.784 & 0.737 \\
1.33 & 1.568 & 0.518 & 1.997 & 0.783 & 0.750 \\
3 & 1.796 & 0.395 & 1.718 & 0.772 & 0.875 \\
4 & 1.891 & 0.356 & 1.626 & 0.769 & 0.904 \\
\hline
\end{tabular}

Table 2. Dimensionless optimum base diameter, length, heat duty, efficiency, and tip temperature for spines of convex parabolic profile $(m=0.75,1,1.25$, $1.33,3$, and 4 ).

\begin{tabular}{cccccc}
\hline$m$ & $D^{*}$ & $l^{*}$ & $Q^{*}$ & $\eta$ & $\theta_{o}$ \\
\hline 0.75 & 1.734 & 0.847 & 2.431 & 0.790 & 0.494 \\
1 & 1.798 & 0.788 & 2.318 & 0.781 & 0.583 \\
1.25 & 1.855 & 0.740 & 2.231 & 0.776 & 0.646 \\
1.33 & 1.872 & 0.727 & 2.207 & 0.774 & 0.663 \\
3 & 2.136 & 0.558 & 1.901 & 0.761 & 0.829 \\
4 & 2.248 & 0.504 & 1.800 & 0.759 & 0.868 \\
\hline
\end{tabular}

for some very common heat transfer modes. Tables 1-4 indicates the optimum values for $n=0,0.5,1$, and 2 . The data given in these tables are identical to the works of Sonn and BarrCohen [3] and Chung et al. [5] for some special cases of $m=1$ (convective fin) and the recent results [6] of $m=4$ (radiative fin). It is noted that the optimum $\theta_{o}$ is omitted in Table 4 because the the finding of Yeh and Liaw [16]. Since inefficient portions begin to show up on fin end, the optimum conditions of a spine does not exist. Thus, for a spine with concave parabolic profile, there is no point found with which slope is equal to $-1 / 5$ when $m$ is less than 0.3 .

To save the time for calcualtion and to minimize the reading errors, the exact design values are provided 
Table 3. Dimensionless optimum base diameter, length, heat duty, efficiency, and tip temperature of conical spines for $m=0.75,1,1.25,1.33,3$, and 4 .

\begin{tabular}{cccccc}
\hline$m$ & $D^{*}$ & $l^{*}$ & $Q^{*}$ & $\eta$ & $\theta_{o}$ \\
\hline 0.75 & 1.889 & 1.701 & 2.483 & 0.782 & 0.293 \\
1 & 1.954 & 1.001 & 2.370 & 0.772 & 0.409 \\
1.25 & 2.012 & 0.943 & 2.283 & 0.766 & 0.493 \\
1.33 & 2.030 & 0.927 & 2.258 & 0.764 & 0.516 \\
3 & 2.309 & 0.716 & 1.949 & 0.750 & 0.750 \\
4 & 2.429 & 0.648 & 1.846 & 0.747 & 0.806 \\
\hline
\end{tabular}

Table 4. Dimensionless optimum base diameter, length, heat duty, and efficiency for spines of concave parabolic profile ( $m=0.75,1,1.25,1.33,3$, and 4$)$.

\begin{tabular}{ccccc}
\hline$m$ & $D^{*}$ & $l^{*}$ & $Q^{*}$ & $\eta$ \\
\hline 0.75 & 2.031 & 1.543 & 2.493 & 0.759 \\
1 & 2.097 & 1.448 & 2.385 & 0.750 \\
1.25 & 2.157 & 1.369 & 2.299 & 0.744 \\
1.33 & 2.175 & 1.346 & 2.275 & 0.742 \\
3 & 2.467 & 1.046 & 1.968 & 0.728 \\
4 & 2.593 & 0.947 & 1.864 & 0.725 \\
\hline
\end{tabular}

concave-parabolic fin only exists for all zero $\theta_{o}$. Also, from Eq. (10), it is observed that the optimum heat transfer is proportional to three-fifth power of fin volume. If the heat duty from a single spine is doubled, an increase of 3.175 times in volume is needed. Thus, it is better to have larger numbers of smaller fins than to have fewer numbers of larger fins from the heat transfer point of view.

\section{CONCLUDING REMARKS}

For given cylindrical, convex parabolic, conical, and concave parabolic fin profiles, the optimum dimensions and heat transfer characteristics of spines for various heat transfer modes are obtained with the aid of fin parameter and fin efficiency. It turns out that the optimum base diameter, length, and heat duty are mainly a function of fin volume, base heat transfer coefficient, and thermal conductivity of a spine. For all the optimum spines, the fin efficiency in convection is higher that that in boiling or radiating heat transfer modes. In addition, it is found that no optimum dimensions of conical and concave-parabolic fins exist for $m$ close to zero. All the optimum data are given nondimensionally in tables as well as charts for convenience of thermal design of heat transfer components.

\section{NOMENCLATURE}

$D \quad$ base diameter of a spine, $\mathrm{m}$

$f$ dimensionless temperature, $\theta / \theta_{b}$

$h$

$k$

$l$

$m$

$N$

$n$

$Q$

$q$

V

$X$

$x$

$\epsilon$

$\eta$

$\theta$

$\sigma$

\section{Subscripts and superscripts}

a ambient

b fin base

o fin tip

opt optimum

* nondimensional quantity

\section{REFERENCES}

1. Kern, D.Q., and Kraus, A.D., Extended surface heat transfer, McGraw Hill, New York, 1972.

2. Kraus, A. D., "Sixty-five Years of Extended Surface Technology (1922-1987),"Appl. Mech. Rev., Vol.41, No. 9, pp. 321-364, 1988.

3. Sonn, A., and Bar-Cohen, A., "Optimum Cylindrical Pin Fin," Journal of Heat Transfer, Vol. 103, p. 814, 1981.

4. Li, C.H., "Optimum Cylindrical Pin Fins," AIChE Journal, Vol. 29, pp. 1043-1044, 1983.

5. Chung, B.T.F., Talbot, D.J., and Van Dyke, J.M., "A New Look at the Optimum Dimensions of Convective Spines," AIChE Symposium Series, Vol. 84, pp. 108113, 1988.

6. Chung, B.T.F., and Nguyen, J. R., "Thermal Analysis and Optimum Design for Radiating Spine of Various Geometries, Heat Transfer Science and Technology (Edited by B.X. Wang), Hemisphere Publishing Corp., pp. 510-517, 1987.

7. Haley, K.W., and Westwater, J.W., "Boiling Heat Transfer from Single Fins," Proc. 3rd Int. Heat Transfer Conf., Vol. 13, pp. 245-253, 1966.

8. Yeh, R.H. and Liaw, S.P., "Optimum Configuration of a Fin for Boiling Heat Transfer," J. of the Franklin Institute, Vol. 330, pp. 153-163, 1993.

9. Razani, A., and Zohoor, H., "Optimum Dimensions of Convecr tive Radiative Spines Using a Temper- 
ature Correlated Profile $J$. of the Franklin Institute, Vol. 328, pp. 471-486, 1991.

10. Sohrabpour, S., and Razani, A., "Optimum Dimensions of Convective Fin with Temperaturer Dependent Thermal Parameters,"J. of the Franklin Institute, Vol. 330, pp. 37-49, 1993.

11. Chung, B.T.F., and Iyer, J.R., "Optimum Design of Longitudinal Rectangular Fins and Cylindrical Spines with Variable Heat Transfer Coefficient," heat transfer engineering, Vol. 14, pp. 31-42, 1993.

12. Patankar, S.V., Numerical Heat Transfer and Fluid Flow, Hemisphere, Washington, DC, p. 48, 1980.

13. Wilkins, J.E. Jr., "Minimum Mass Thin Fins Which
Transfer Heat Only by Radiation to Surrounding at Absolute Zero", J. Soc. Ind. Appl. Math., Vol. 8, pp. 630-639, 1960.

14. Dhir, V. K., and Liaw, S. P., "Framework for a Unified Model for Nucleate and Transition Boiling," Journal of Heat Transfer, Vol. 111, pp. 739-746, 1989.

15. Gaertner, R.F., "Photographic Study of Nucleate Pool Boiling on a Horizontal Surface," Journal of Heat Transfer, Vol. 87, pp. 17-29, 1965.

16. Yeh, R.H., and Liaw, S.P., "An Exact Solution for Thermal Characteristics of Fins with PowerLaw Heat Transfer Coeffricient," Int. Comm. Heat Mass Transfer, Vol. 17, pp. 317-330, 1990. 